\title{
Combination of Procyanidins Extracted from Lotus Seedpod and $N$-acetyl Cysteine Ameliorates Scopolamine-induced Memory Impairment in Mice
}

\author{
Yong Sui ${ }^{1,2}$, Juan Xiao ${ }^{3}$, Shuyi $\mathrm{Li}^{4}$, Xiaopeng $\mathrm{Li}^{1,2}$, Bijun Xie ${ }^{1}$, Zhida Sun ${ }^{1, *}$ \\ ${ }^{1}$ College of Food Science and Technology, Huazhong Agricultural University, Wuhan, China \\ ${ }^{2}$ Key Laboratory of Environment Correlative Dietology, Ministry of Education, Huazhong Agricultural University, Wuhan, China \\ ${ }^{3}$ Key Laboratory of Functional Foods, Ministry of Agriculture/Guangdong Key Laboratory of Agricultural Products Processing, \\ Sericultural \& Agri-food Research Institute, Guangdong Academy of Agricultural Sciences, Guangzhou, China \\ ${ }^{4}$ College of Food Science and Engineering, Wuhan Polytechnic University, Wuhan, China \\ *Corresponding author: sunzhida@sina.com
}

Received August 13, 2015; Revised August 25, 2015; Accepted September 10, 2015

\begin{abstract}
The main purpose of this study is to determine the effect of combined procyanidins extracted from the lotus seedpod (LSPC) and $N$-acetyl cysteine (NAC) on the memory impairment induced by scopolamine (3 mg/kg, i.p.) in mice. The capacities of memory and learning were evaluated by Y-maze test after 20-day administration of NAC (90 mg/kg BW), LSPC (60 mg/kg BW), and combined LSPC and NAC (60 mg/kg BW + $90 \mathrm{mg} / \mathrm{kg} \mathrm{BW),}$ respectively. It was indicated that LSPC and combined LSPC and NAC significantly reduced the number of incorrect responses compared with that of scopolamine in Y-maze test. In addition, combination of LSPC and NAC was also found to enhance total antioxidant capacity (T-AOC) level, superoxide dismutase (SOD) and glutathione peroxidase (GPx) activities in brain and serum, and inhibit acetyl cholinesterase (AchE) activity in brain and neural nitric oxide synthase (nNOS) activities as well as nNOS relative mRNA level. To be mentioned, the T-AOC level in brain of LSPC+NAC group increased by $32.46 \%$ and $36.04 \%$ relative to those of NAC group and LSPC group, respectively, and the GPx activity increased by $45.79 \%$ and $16.17 \%$, while the nNOS activity decreased by $38.24 \%$ and $36.36 \%$. These results demonstrate that combination of LSPC and NAC exhibited better ameliorative effects in scopolamine-induced memory impairment mice than treated by NAC and LSPC alone, which suggest LSPC and NAC combination may provide a viable therapy in the treatment for Alzheimer's disease and other forms of cognitive impairment diseases.
\end{abstract}

Keywords: procyanidins extracted from lotus seedpod (LSPC), N-acetyl cysteine (NAC), scopolamine, memory impairment, antioxidant activity

Cite This Article: Yong Sui, Juan Xiao, Shuyi Li, Xiaopeng Li, Bijun Xie, and Zhida Sun, "Combination of Procyanidins Extracted from Lotus Seedpod and $N$-acetyl Cysteine Ameliorates Scopolamine-induced Memory Impairment in Mice.” Journal of Food and Nutrition Research, vol. 3, no. 7 (2015): 464-470. doi: 10.12691/jfnr-3-7-8.

\section{Introduction}

Alzheimer's disease (AD) is a typical neurodegenerative disorder characterized by progressive cognitive and memory deficit, and affects more and more people due to the increase in life expectancy these days [1,2,3]. Although the mechanisms of AD is complex and still remain to be clearly illuminated, many studies have indicated that oxidative stress and overexpressing nitric oxide synthase are closely associated to learning and memory impairment in aged persons as well as $A D$ patients $[4,5,6,7]$. A great deal of evidence demonstrated the importance of oxidative stress and amyloid- $\beta(A \beta)$ protein formation and accumulation in $\mathrm{AD}$ pathogenesis, and therefore might play a key role [4,8]. Oxidative stress was evident in $\mathrm{AD}$ brain by markedly increased levels of protein [8,9], lipid, DNA, and RNA oxidation, which established oxidative stress as an early event in $\mathrm{AD}$ pathogenesis that could be treated as a therapeutic target $[10,11]$. The increased oxidative damage in the aging process might be resulted from the deficiency of antioxidants. Valko et al. [4] and Lee et al. [12] show clearly that administration with antioxidants can noticeably reverse these alterations. Currently, tacrine and rivastigmine are the only ones available by prescription for the treatment of $\mathrm{AD}$, but they are not the ideal drugs because of those common side effects such as hepatotoxicity and nausea and vomiting [13]. Therefore, it is well worth seeking effective antioxidants with low or none toxicity for the therapy of $\mathrm{AD}$ with no damage to the body.

Increasing researches demonstrated that the ability of phenolic rich plant extract to significantly enhance the antioxidant efficiency of synthetic antioxidants in vitro. The use of combined antioxidants, called hurdle technology, has been accepted and applied to food preservation [14,15], but synergistic interaction of 
combined antioxidants for ameliorate memory impairment in vivo is still poorly explored. The mixture of catechin and epicatechin significantly reversed the $A \beta$-induced neuronal cell death and showed the highest protective effect and notable synergistic activity [16]. The combined treatment of memantine (an $N$-Methyl-D-aspartate receptor antagonist) and tea polyphenol was more effective in neuroprotection than either alone in mice excitotoxic injury [17]. Melatonin and resveratrol exhibited neuroprotection against oxidative stress in rat primary neurons and astrocytes by stability control of heme oxygenase- 1 protein via regulation of PI3K/Akt-GSK3 $\beta$ pathways. The synergistic neuroprotective activity of coadministration of melatonin and resveratrol may provide another way to inhibit neurodegeneration while decreasing the adverse side effects when using single compounds under high concentrations [18]. For this reason, it is necessary to evaluate the effect of combined antioxidants to reduce the learning and memory impairment in aged persons as well as AD patients.

Procyanidins are ubiquitous and present as the second most abundant group of natural phenolics after lignin, widely distributed in fruit, vegetables, cereals, cocoa and wine $[19,20]$. As the inedible part of Nelumbo nucifera Gaertn (Nymphaeaceae), lotus seedpod is used as a traditional Chinese herbal medicine with hemostatic function [21]. Our laboratory found that lotus seedpod was rich in procyanidins, and procyanidins extract from lotus seedpod (LSPC) was characterized for the first time. Meanwhile we attested that LSPC had the potent antioxidant activity in vitro and in vivo, and the ameliorative effect for oxidative damage and cognitive disorder in memory impairment mice and aged rats [22,23]. $N$-acetyl cysteine (NAC) is the precursor of glutathione that plays a critical role in oxidative stress [24]. Increasing studies demonstrated that NAC exhibited the protective effect on learning and memory deficit rats induced by 2cyclohexene-1-one and A $\beta$-peptide [25,26]. Actually, both LSPC and NAC can freely cross the blood-brain barrier and be detected in the brain [24,27]. However, the ameliorative effects of combined LSPC and NAC on memory impairment in mice have not been established.

The present study tried to evaluate the ameliorative effects of combined LSPC and NAC on learning and memory ability in scopolamine-induced mice. Moreover, alterations in antioxidant ability of brain and serum, brain cholinergic activity, and nNOS activity as well as relative mRNA level of brain were determined as the possible mechanisms.

\section{Materials and Methods}

\subsection{Chemicals and Reagents}

$\mathrm{N}$-acetyl cysteine (NAC) was purchased from Simopharm Chemical Reagent CO., LtD. (Shanghai, China). Piracetam was obtained from Hubei Huazhong Pharma.CO., LtD. (Wuhan, China). Scopolamine hydrobromide injection was purchased from Xuzhou RYEN Pharma.CO., LtD. (Xuzhou, China). Kits for determination of acetylcholinesterase (AchE), total antioxidant capacity (T-AOC), superoxide dismutase (SOD), glutathione peroxidase (GPx), total nitric oxide synthase (tNOS), inducible nitric oxide synthase (iNOS), neural nitric oxide synthase (nNOS) and protein levels were purchased from Nanjing Jiancheng Bioengineering Institute. (Nanjing, China).

\subsection{Preparation of LSPC}

Fresh lotus seedpods of Nelumbo Nucifera Gaertn (cultivar: Number 2 Wuhan plant) were harvested from Honghu (Hubei, China) in late July 2011 and quickly stored at $-20^{\circ} \mathrm{C}$ until use. This variety of lotus was identified by Prof. Xueming Ni from Department of Botany, Wuhan Plant Institute of the Chinese Academy of Science [22]. The extraction method of lotus seedpod procyanidins (LSPC) was according to a previous article [21]. The purity of obtained LSPC with $98.9 \%$ was measured by the method reported by Porter et al. [28]. And LSPC were stored in a freezer at $-20^{\circ} \mathrm{C}$. Electrospray ionization mass spectrometry analysis has revealed that LSPC mainly contains monomers, and B-type dimers, trimers as well as tetramers, and the base units of LSPC oligomers are catechin and epicatechin [29]. The percentage of catechin, epicatechin, dimers, trimers and tetramers in LSPC is $10.9 \%, 9.1 \%, 53.6 \%, 19.5 \%$ and $1.9 \%$, respectively.

\subsection{Animals}

Male Kunming mice (20 \pm 2 g) were purchased from Wuhan University Research Center for Animal Experiment (Hubei, China). A total of 60 mice were used in the experiments and all experimental procedures were carried out at Medical Experiment Department of Wuhan General Hospital of Guangzhou Military Command (Hubei, China). During the experiment, animals were housed five per cage and kept at a controlled temperature $25 \pm 1{ }^{\circ} \mathrm{C}$, humidity $55-60 \%$ and $12 \mathrm{~h}$ light/12 h dark daily cycle with access normal solid diet and top water ad libitum. All the mice were cared for strictly according to the Guiding Principles in the Care and Use of Animals. The experiment was approved by the ethics regulations of Animal Care Committee of Wuhan General Hospital of Guangzhou Military Command and Huazhong Agricultural University (Hubei, China).

\subsection{Treatments}

After adaptation for 3 days, the animals were randomly divided into six groups with ten mice in each group: control (CON), scopolamine control (SCOP), positive drug control (Piracetam), $N$-acetyl cysteine group (NAC), LSPC group (LSPC), and combined LSPC and $N$-acetyl cysteine group (LSPC+NAC). Mice in CON, SCOP, Piracetam, NAC, LSPC and LSPC+NAC groups were given distilled water, distilled water, piracetam (400 $\mathrm{mg} / \mathrm{kg} \mathrm{BW}$ ), $N$-acetyl cysteine (90 mg/kg BW), LSPC (60 $\mathrm{mg} / \mathrm{kg} \mathrm{BW}$ ) and a mixture of LSPC (60 mg/kg BW) and $\mathrm{N}$-acetyl cysteine (90 mg/kg BW) at a dose of $0.1 \mathrm{~mL} / 10 \mathrm{~g}$ body weight (BW) by oral gavage once daily for 20 days, respectively. The dosage was based on previous experimental work $[3,30]$.

Solutions of Piracetam $(40 \mathrm{mg} / \mathrm{mL})$, NAC $(9 \mathrm{mg} / \mathrm{mL})$ and LSPC $(6 \mathrm{mg} / \mathrm{mL})$ were prepared in distilled water. Solutions of combined LSPC and NAC were composed of $6 \mathrm{mg} / \mathrm{mL}$ LSPC and 9mg/mL NAC. All solutions were freshly prepared every day and administrations began at 
9:00 a.m. At the $20^{\text {th }}$ day, animals except CON group were administrated with scopolamine ( $3 \mathrm{mg} / \mathrm{kg} \mathrm{BW}$, i.p.) to induce memory impairment $30 \mathrm{~min}$ before a training course of Y-maze test. Animals in CON group were treated with $0.9 \%$ saline (i.p.) in the same way.

\subsection{Y-maze Test}

To assess the learning and memory abilities of the mice, a Y-maze test was carried out at the end of the treatment experiment as described previously [22,30]. Briefly, the Y-maze consisted of three arms $(30 \mathrm{~cm} \times 15 \mathrm{~cm} \times 10 \mathrm{~cm})$ with a lamp at the end of each arm and the bottom of each arm covered with electric net. The bottom of the arm was not covered with electric current when the lamp of this arm was turned on, taken as safe area. At this moment, the other two arms were covered with electric current (50-70V) and without lamp turned on, taken as unsafe area. At the beginning, each animal was placed separately at the end of one arm to adjust for 5 min without electric shock. Then one lamp was turned on and electric shock compelled the mouse to move to the safe arm, where the mouse adjusted to the condition for $30 \mathrm{sec}$; and then another electric shock was switched following the sequence $\mathrm{ABCCAB}$ (A, B, C represent three arms of Y-maze ) to force the mouse to move to the safe arm. Such trial was repeated 35 times during the training process and repeated 10 times after 24 $\mathrm{h}$ during the test process. During the experiment, it would be a correct response if the mouse directly moved to the safe arm within $30 \mathrm{sec}$; otherwise it would be an incorrect response. The incorrect response was recorded in the test process to reflect the learning and memory ability of the mouse. The less the incorrect responses, the better the learning and memory ability of the mouse was.

\subsection{Determination of T-AOC level, and AchE, SOD, GSH-Px, tNOS, iNOS and nNOS activities}

After Y-maze test, blood of each mouse was obtained from the tail veins. Then all the mice were killed by cervical dislocation under anesthesia. The brains were immediately dissected and washed with ice-cold normal saline. Five right brain samples in each group were randomly selected and frozen in liquid nitrogen and stored at $-80{ }^{\circ} \mathrm{C}$ for real-time PCR. The rest brain samples were weighed and homogenized with a $50 \mathrm{mmol} / \mathrm{L}$ phosphate buffer (pH 7.0) containing $0.1 \mathrm{mmol} / \mathrm{L}$ EDTA before use. Brain homogenate was centrifuged at 4000rmp for $10 \mathrm{~min}$ at $4{ }^{\circ} \mathrm{C}$ and the supernatant was used for AchE , T-AOC, SOD, GPx, tNOS, iNOS and nNOS tests. Blood was centrifuged at $4000 \mathrm{rmp}$ for $10 \mathrm{~min}$ at $4{ }^{\circ} \mathrm{C}$ to obtain serum for T-AOC, SOD and GPx tests. All parameters were determined using the respective kits according to the manufacturer's specifications.

\subsection{Quantitative Real-Time PCR}

For nNOS, total RNA of the right brain was isolated using Trizol reagent (15596-026, Invitrogen) as directed by the manufacturer's specifications, and reversetranscribed using a RevertAid First Strand cDNA Synthesis Kit (K-1622, Fermentas) at $42{ }^{\circ} \mathrm{C}$ for $30 \mathrm{~min}$ followed by $80{ }^{\circ} \mathrm{C}$ for $5 \mathrm{~min}$. Real-time polymerase chain reaction was conducted with Thunderbird SYBR qPCR
Mix (QPS-201, Toyobo Biologics), using the real-time thermocycler (Hangzhou Bioer Technology Co., LtD), and the cycle conditions were $95{ }^{\circ} \mathrm{C}$ for $60 \mathrm{sec}$ and 40 cycles of $95{ }^{\circ} \mathrm{C}$ for $15 \mathrm{sec}$ and $72{ }^{\circ} \mathrm{C}$ for 20 sec. The dissociation curve of each gene was conducted and analyzed using the SLAN Quantitative Real-Time PCR detection system (Shanghai Hongshi Medical Technology Co., Ltd), and the result confirmed the specificity of the product. Each sample was conducted in triplicate and normalized to $\beta$ actin. The relative expression levels of the genes were analyzed with the $2^{-\Delta \Delta}{ }^{\mathrm{CT}}$ method as previously described [31]. The sequences of the primers (Invitrogen) for the genes were as follows: nNOS, forward 5'GCTTCAGGAATATGAGGAATGG-3', reverse 5'TGATGGAATAGTAGCGAGGTTGT-3'; $\quad \beta$-actin, forward 5'-CTGAGAGGGAAATCGTGCGT-3', reverse5'CCACAGGATTCCATACCCAAGA-3'.

\subsection{Statistical Analysis}

Data were expressed as mean \pm standard deviation (SD). All data were analyzed using a one-way ANOVA, followed by Duncan post hoc test if difference was significant $(P<0.05)$ in groups. Statistical analyses were conducted by the SPSS 16.0 software, and a difference was regarded significant when $\mathrm{P}<0.05$.

\section{Results}

\subsection{The Effect of Combined LSPC and NAC on $Y$-maze Test}

Effects of combined LSPC and NAC on Y-maze test in scopolamine-induced memory impairment model were shown in Figure 1. SCOP group exhibited obviously increased number of incorrect responses in Y-maze test in comparison with CON group $(\mathrm{P}<0.05)$. However, incorrect responses were significantly decreased by treatments with Piracetam, NAC, LSPC and combined LSPC and NAC as compared to SCOP group $(\mathrm{P}<0.05)$. Moreover, incorrect responses of animals in LSPC+NAC group in comparison with SCOP group and NAC group were reduced by $50.55 \%$ and $34.11 \%$, respectively, but showing no significant difference relative to CON group and LSPC group.

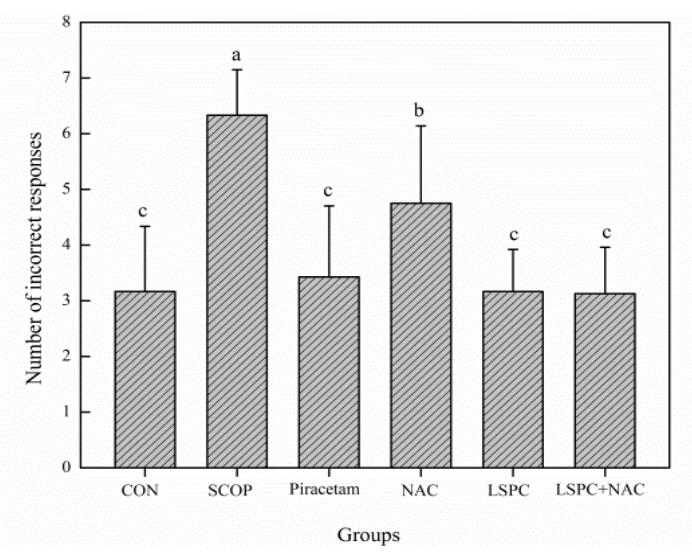

Figure 1. Effects of combined LSPC and NAC on number of incorrect responses of Y-maze test in scopolamine-induced memory impairment mice. Bars represent the Mean \pm SD in each group $(n=10)$. Groups with different letters ( $\mathrm{a}, \mathrm{b}, \mathrm{c})$ above the bars are significantly different $(\mathrm{P}<$ 0.05), while sharing any same letters mean insignificant differences 


\subsection{The Effect of Combined LSPC and NAC on Antioxidant Capacity and AchE Activity in Brain}

In scopolamine-induced memory impairment model, ANOVA indicated that obviously decreased T-AOC level, SOD and GPx activities, and the ratio of GPX to SOD (GPx/SOD) of brain in SCOP group were observed when compared with CON group ( $\mathrm{P}<0.05$, Table 1$)$. However, treatments with Piracetam, NAC, LSPC and combined LSPC and NAC significantly reversed those declines in comparing with SCOP group $(\mathrm{P}<0.05)$, the SOD and GPx activities and the ratio of GPx/SOD reaching to the level of equal or superior to CON group. Combined LSPC and NAC treatment exhibited higher SOD activity than the single LSPC and NAC treatments, but without significant differences. Importantly, T-AOC level, GPx activities and the ratio of $\mathrm{GPx} / \mathrm{SOD}$ in $\mathrm{LSPC}+\mathrm{NAC}$ group were significantly better than those in NAC group and LSPC group $(\mathrm{P}<0.05)$. T-AOC level in LSPC+NAC group increased by $32.46 \%$ and $36.04 \%$ in comparison with NAC group and LSPC group, respectively, and GPx activity increased by $45.79 \%$ and $16.17 \%$, respectively.

ANOVA indicated that the AchE activity in scopolamine-treated mice brains was obviously higher than that in CON group $(\mathrm{P}<0.05$, Table 1$)$. AchE activity in Piracetam, NAC, LSPC and LSPC+NAC groups was obviously weaker than that in SCOP group ( $\mathrm{P}<0.05)$, but without significant differences among those groups.

Table 1. Effects of combined LSPC and NAC on the level of T-AOC, the activity of SOD and GPx and AchE, and the ratio of GPx/SOD in brains of scopolamine-induced memory impairment mice

\begin{tabular}{|c|c|c|c|c|c|}
\hline Group & $\begin{array}{c}\text { T-AOC } \\
\text { (U/mgprot) }\end{array}$ & $\begin{array}{c}\text { SOD } \\
\text { (U/mgprot) }\end{array}$ & $\begin{array}{c}\text { GPX } \\
\text { (U/mgprot) }\end{array}$ & GPx/ SOD & $\begin{array}{c}\text { AchE } \\
\text { (U/mgprot) }\end{array}$ \\
\hline $\mathrm{CON}$ & $1.25 \pm 0.10^{\mathrm{c}}$ & $113.36 \pm 7.75^{b}$ & $53.06 \pm 4.25^{b}$ & $0.47 \pm 0.06^{\mathrm{bc}}$ & $0.47 \pm 0.05^{\mathrm{b}}$ \\
\hline SCOP & $0.84 \pm 0.10^{\mathrm{a}}$ & $102.72 \pm 2.31^{\mathrm{a}}$ & $42.55 \pm 4.49^{\mathrm{a}}$ & $0.41 \pm 0.04^{\mathrm{a}}$ & $0.58 \pm 0.07^{\mathrm{a}}$ \\
\hline Piracetam & $1.11 \pm 0.06^{\mathrm{b}}$ & $119.98 \pm 5.04^{\mathrm{bc}}$ & $62.85 \pm 2.50^{c}$ & $0.52 \pm 0.03^{\mathrm{cd}}$ & $0.48 \pm 0.09^{b}$ \\
\hline NAC & $1.14 \pm 0.07^{\mathrm{bc}}$ & $112.28 \pm 5.37^{\mathrm{b}}$ & $48.94 \pm 6.83^{b}$ & $0.44 \pm 0.05^{\mathrm{ab}}$ & $0.48 \pm 0.06^{\mathrm{b}}$ \\
\hline LSPC & $1.11 \pm 0.11^{\mathrm{b}}$ & $119.86 \pm 7.96^{\mathrm{bc}}$ & $61.42 \pm 3.41^{c}$ & $0.51 \pm 0.04^{\mathrm{c}}$ & $0.44 \pm 0.05^{\mathrm{b}}$ \\
\hline $\mathrm{LSPC}+\mathrm{NAC}$ & $1.51 \pm 0.06^{\mathrm{d}}$ & $125.05 \pm 10.94^{c}$ & $71.35 \pm 9.44^{\mathrm{d}}$ & $0.57 \pm 0.03^{\mathrm{d}}$ & $0.46 \pm 0.06^{\mathrm{b}}$ \\
\hline
\end{tabular}

Values are presented as mean \pm SD in each group $(n=10)$. Means in the same column with different superscripts $(\mathrm{a}, \mathrm{b}$, c, $\mathrm{d})$ are significantly different $(\mathrm{P}<0.05)$, while sharing any same letters mean insignificant differences.

\subsection{The Effect of Combined LSPC and NAC on Antioxidant Capacity in Serum}

As shown in Table 2, T-AOC level, SOD and GPX activities in scopolamine-treated mice serum were significantly lower than that in CON group $(\mathrm{P}<0.05)$. However, treatments with Piracetam, NAC, LSPC and combined LSPC and NAC significantly prevented those declines in comparing with SCOP group $(\mathrm{P}<0.05)$, the T-
AOC level and SOD and GPx activities and the ratio of GPX/SOD reaching to the level of equal or superior to CON group. Combined LSPC and NAC treatment exhibited higher SOD and GPx activities than the single LSPC and NAC treatments, but no significant difference was observed among all above groups. T-AOC level in serum of LSPC+NAC group increased by $11.36 \%$ and $15.44 \%$ relative to those of NAC group and LSPC group, respectively.

Table 2. Effects of combined LSPC and NAC on the level of T-AOC, the activity of SOD and GPx, and the ratio of GPx/SOD in serum of scopolamine-induced memory impairment mice

\begin{tabular}{|c|c|c|c|c|}
\hline Group & $\begin{array}{c}\text { T-AOC } \\
\text { (U/mgprot) }\end{array}$ & $\begin{array}{c}\text { SOD } \\
\text { (U/mgprot) }\end{array}$ & $\begin{array}{c}\text { GPx } \\
\text { (U/mgprot) }\end{array}$ & GPx/SOD \\
\hline CON & $5.26 \pm 0.41^{\mathrm{b}}$ & $195.28 \pm 12.84^{\mathrm{b}}$ & $580.75 \pm 27.25^{\mathrm{bc}}$ & $2.97 \pm 0.22^{\mathrm{ab}}$ \\
\hline SCOP & $4.67 \pm 0.28^{\mathrm{a}}$ & $172.84 \pm 16.08^{\mathrm{a}}$ & $530.87 \pm 25.30^{\mathrm{a}}$ & $2.79 \pm 0.28^{\mathrm{a}}$ \\
\hline Piracetam & $5.20 \pm 0.24^{b}$ & $202.19 \pm 13.55^{b}$ & $605.33 \pm 11.22^{d}$ & $3.00 \pm 0.19^{\mathrm{ab}}$ \\
\hline NAC & $5.37 \pm 0.26^{b}$ & $191.09 \pm 19.13^{b}$ & $562.70 \pm 25.21^{b}$ & $2.96 \pm 0.32^{\mathrm{ab}}$ \\
\hline LSPC & $5.18 \pm 0.19^{b}$ & $190.11 \pm 16.23^{\mathrm{b}}$ & $593.22 \pm 14.38^{\mathrm{cd}}$ & $3.14 \pm 0.33^{\mathrm{b}}$ \\
\hline LSPC+NAC & $5.98 \pm 0.29^{c}$ & $193.72 \pm 15.10^{\mathrm{b}}$ & $604.51 \pm 17.69^{d}$ & $3.11 \pm 0.28^{b}$ \\
\hline
\end{tabular}

Values are presented as mean \pm SD in each group $(n=10)$. Means in the same column with different superscripts $(a$, b, c, d) are significantly different $(\mathrm{P}<0.05)$, while sharing any same letters mean insignificant differences.

\subsection{The Effect of Combined LSPC and NAC on tNOS, iNOS and nNOS Activities and nNOS mRNA Level in Brain}

As shown in Table 3, scopolamine resulted in visible enhancements in tNOS and nNOS activities in brain as compared to CON group $(\mathrm{P}<0.05)$. However, treatments with Piracetam, NAC, LSPC and combined LSPC and NAC significantly reversed those increases in comparing with SCOP group $(\mathrm{P}<0.05)$, declining to the level of equal or inferior to CON group. Importantly, the activity of tNOS and nNOS in LSPC+NAC group were significantly lower than those in NAC group and LSPC group $(\mathrm{P}<0.05)$. The tNOS activity in LSPC+NAC group decreased by $25.53 \%$ and $27.08 \%$ relative to those of NAC group and LSPC group, respectively, nNOS activity decreased by $38.24 \%$ and $36.36 \%$, respectively. But there was no evident alteration in iNOS activity in brain to all the experimental groups. 
Table 3. Effects of combined LSPC and NAC on the activity of tNOS, iNOS and nNOS in brains of scopolamine-induced memory impairment mice

\begin{tabular}{|c|c|c|c|}
\hline Group & $\begin{array}{c}\text { tNOS } \\
\text { (U/mgprot) }\end{array}$ & $\begin{array}{c}\text { iNOS } \\
\text { (U/mgprot) }\end{array}$ & $\begin{array}{c}\text { nNOS } \\
\text { (U/mgprot) }\end{array}$ \\
\hline CON & $0.48 \pm 0.06^{\mathrm{b}}$ & $0.14 \pm 0.02$ & $0.34 \pm 0.07^{\mathrm{b}}$ \\
\hline SCOP & $0.60 \pm 0.05^{\mathrm{a}}$ & $0.15 \pm 0.02$ & $0.45 \pm 0.05^{\mathrm{a}}$ \\
\hline Piracetam & $0.48 \pm 0.04^{\mathrm{b}}$ & $0.15 \pm 0.02$ & $0.33 \pm 0.04^{\mathrm{b}}$ \\
\hline NAC & $0.47 \pm 0.04^{\mathrm{b}}$ & $0.13 \pm 0.02$ & $0.34 \pm 0.03^{\mathrm{b}}$ \\
\hline LSPC & $0.48 \pm 0.06^{\mathrm{b}}$ & $0.15 \pm 0.02$ & $0.33 \pm 0.07^{\mathrm{b}}$ \\
\hline LSPC+NAC & $0.35 \pm 0.09^{\mathrm{c}}$ & $0.14 \pm 0.03$ & $0.21 \pm 0.08^{\mathrm{c}}$ \\
\hline
\end{tabular}

Values are presented as mean \pm SD in each group $(\mathrm{n}=10)$. Means in the same column with different superscripts (a, b, c) are significantly different $(P<0.05)$, while sharing any same letters mean insignificant differences.

The change tend of nNOS activity and relative mRNA level in brains were similar to all therapeutic groups. As shown in Figure 2, SCOP group exhibited significantly higher nNOS relative mRNA level than CON group. Nevertheless, after treatments with Piracetam, NAC, LSPC and combined LSPC and NAC those increases in mice brains were significantly reversed in comparison with SCOP group $(\mathrm{P}<0.05)$. Compared with both NAC group and LSPC group, LSPC+NAC group showed marked decrease in the nNOS relative mRNA level $(\mathrm{P}<$ 0.05).

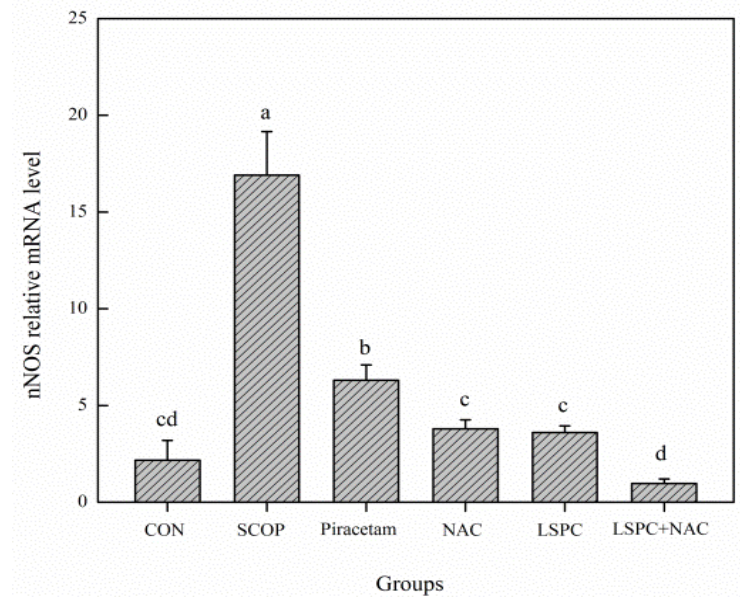

Figure 2. Effects of combined LSPC and NAC on the mRNA level of nNOS in brains of scopolamine-induced memory impairment mice. Bars represent the Mean \pm SD in each group $(n=10)$. Groups with different letters (a, b, c, d) above the bars are significantly different $(\mathrm{P}<0.05)$, while sharing any same letters mean insignificant differences

\section{Discussion}

In our study, the effect of ameliorating learning and memory deficits of LSPC and NAC was assessed in a mouse model of memory impairment induced by scopolamine. Scopolamine is a muscarinic receptor antagonist, which can impair learning and memory ability to some degree [32]. In animal models of impaired learning and memory, scopolamine has been widely employed to evaluate the potential memory-enhancing agent $[3,33]$. In this study, scopolamine resulted in bad performance in Y-maze compared with the CON group. These results confirmed marked memory deterioration in mice, and memory was closely related to central cholinergic system, which accorded with the previous researches [30]. However, LSPC+NAC group were able to ameliorate memory injury induced by scopolamine by significantly declining the number of incorrect responses. The incorrect responses of LSPC+NAC group were decreased by $34.11 \%$ relative to those of NAC group, which indicated that combined LSPC and NAC was more effective than NAC to reduce scopolamine-induced memory impairment, suggesting LSPC and NAC combination had a memory-enhancing activity.

As reported, scopolamine can impair learning and memory processing in animals caused by cholinergic deficits and oxidative damage [34,35]. As an important neurotransmitter in brain, acetylcholine (ACh) plays a key role in memory and learning processes [36]. It is well known that choline acetyltransferase (ChAT) is the acetylcholine synthesis enzyme and AChE is responsible for hydrolyzing acetylcholine. Both ChAT and AChE are the critical enzymes in modulation the metabolism of Ach. Actually, ChAT is not restricting for the rapid synthesis of ACh under normal conditions [37], but AChE plays an important role for the rapid hydrolysis of ACh. Thus, AChE is considered to dominate the ACh level in brain [3]. Currently, the most accepted treatment for memory deficit and $\mathrm{AD}$ is administration of AChE inhibitors for activation of ACh synthesis [13]. In this study, scopolamine not just leaded to more evidently incorrect responses in Y-maze test, but leaded to higher AchE activity compared with CON group, which was in accordance with previous studies [30]. However, combined LSPC and NAC decreased AchE activity to the normal level. Consequently, combined LSPC and NAC improved learning and memory ability in mice induced by scopolamine via declining AChE activity. The memory improving effects of NAC and LSPC alone were consistent with previous researches $[3,26]$.

The age related enhancement in oxidative stress leads to the significant increase in oxidative damage to central nervous system. In the brain, antioxidant defense system performs as a complex team to scavenge free radicals [38]. Oxidative damage is a typical and early feature of $\mathrm{AD}$ and plays a key role in its pathogenesis and progression $[39,40]$. It is the cytotoxic consequence of oxyradical and oxidant formation and the reaction with cellular constituents. At present, oxidative stress act in target for therapy of memory impairment and AD. In this study, scopolamine led to a significant decrease in T-AOC level, SOD and GPx activities, and the ratio of GPx/SOD in mice brain and serum compared with the respective CON groups, which suggested that memory impairment induced by scopolamine was associated with antioxidant defense system [30,41]. It is well known that T-AOC reflects the capacity of nonenzymatic antioxidant defense system. It is worth to emphasize that the antioxidant enzymes (SOD, $\mathrm{GPx}$, and catalase) work as a complex team to remove reactive oxygen species, and a balance among these antioxidant enzymes is considered to be critical in antioxidant defense [23,42]. SOD is responsible for converting superoxide to hydrogen peroxide, then GPx and catalase convert hydrogen peroxide to water [43]. However, overexpressing SOD with a defined activity of GPx and catalase may cause accumulation of hydrogen peroxide, which can also result in oxidative damage. Therefore, the ratios of activities of GPx to SOD and 
catalase to SOD have been considered as important indexes to assess oxidative stress [27]. Increasing evidence (included our study) has confirmed that learning and memory deficits in the scopolamine-induced animal model is associated with enhanced oxidative damage in the brain $[4,41,44]$. However, combined LSPC and NAC prevented the declined activities of SOD and GPx caused by scopolamine, which suggested LSPC and NAC combination had a strong effect on improving brain antioxidant defense system. Noteworthily, T-AOC level in brain of LSPC+NAC group increased by $32.46 \%$ and $36.04 \%$ relative to those of NAC group and LSPC group, respectively, and GPx activity increased by $45.79 \%$ and $16.17 \%$, respectively. These results indicated that in scopolamine-induced model of this study, both T-AOC level and GPx activity were significantly raised by combined LSPC and NAC when compared with LSPC or NAC, which might suggest that LSPC and NAC combination is more effective than LSPC or NAC on improving brain antioxidant defense of mice and confirm that combined contributions of two kinds of antioxidants are more potent than that of a sole antioxidant in modulation of oxidative stress in memory deficit. Similar conclusions have been reported previously, LSPC and Lcysteine combinations and co-treatment of huperzine A and ligustrazine phosphate could notably ameliorate scopolamine-induced memory impairment in mice and rats, respectively $[30,44]$.

Many studies has indicated that NO plays an critical role in the process of learning and memory [45]. There are three isoforms of NOS being responsible for the synthesis of NO, which catalyze the conversion of L-arginine to Lcitrulline and NO. Actually, NO production in the young brain is mainly regulated by nNOS as well as gene and protein expression [31]. It is well known that nNOS is primarily distributed in neurons and overexpressing nNOS activity can lead to neurotoxicity and cell apoptosis $[46,47]$. In the present study, the tNOS activity was evidently increased in SCOP group, which is primarily due to significant nNOS activity enhancement. However, treatments with NAC and LSPC effectively declined the nNOS activity and relative mRNA level to the normal level. Interestingly, the nNOS activity in brain of LSPC+NAC group decreased by $38.24 \%$ and $36.36 \%$ relative to those of NAC group and LSPC group, respectively, which indicated that LSPC and NAC synergistically reduced the nNOS activity and relative mRNA level in memory impairment mice.

\section{Conclusion}

In conclusion, NAC, LSPC, and combined LSPC and NAC ameliorate memory impairment induced by scopolamine in mice. LSPC and NAC combinations exhibited better ameliorative effects than treated by NAC and LSPC alone. This mechanism is maybe due to the big hydrogen bond of sulfhydryl formed by NAC with phenolic hydroxyl group of LSPC, thereby, the antioxidant effect of LSPC was increasing. It is point out that LSPC and NAC similarly had the synergistic antioxidant in vivo, and amelioration of brain cholinergic activity, and inhibition of brain nNOS activity as well as relative mRNA level. These findings suggest that combined LSPC and NAC may have a potential effect on treatment of learning and memory dysfunction in aging process and neurodegenerative diseases such as $\mathrm{AD}$.

\section{Acknowledgments}

This work was supported by National Natural Science Foundation of China (NSFC, No. 31071633).

\section{Statement of Competing Interests}

No conflict of interest exists regarding the submission of this manuscript, and the manuscript was approved by all authors for publication.

\section{References}

[1] Zawia, N.H., Lahiri, D.K. and Cardozo-Pelaez, F., "Epigenetics, oxidative stress, and Alzheimer disease," Free Radical Biology and Medicine, 46 (9). 1241-1249. 2009.

[2] Mattson, M.P., Duan, W., Pedersen, W.A. and Culmsee, C., "Neurodegenerative disorders and ischemic brain diseases," Apoptosis, 6 (1-2). 69-81. 2001.

[3] Xu, J., Rong, S., Xie, B., Sun, Z., Zhang, L., Wu, H., Yao, P., Zhang, Y. and Liu, L., "Procyanidins extracted from the lotus seedpod ameliorate scopolamine-induced memory impairment in Mice," Phytotherapy Research, 23 (12). 1742-1747. 2009.

[4] Valko, M., Leibfritz, D., Moncol, J., Cronin, M.T.D., Mazur, M. and Telser, J., "Free radicals and antioxidants in normal physiological functions and human disease," The International Journal of Biochemistry \& Cell Biology, 39 (1). 44-84. 2007.

[5] Liu, P., Smith, P.F., Appleton, I., Darlington, C.L. and Bilkey, D.K., "Nitric oxide synthase and arginase in the rat hippocampus and the entorhinal, perirhinal, postrhinal, and temporal cortices: Regional variations and age-related changes," Hippocampus, 13 (7). 859-867. 2003.

[6] Zhu, X., Raina, A.K., Lee, H.-g., Casadesus, G., Smith, M.A. and Perry, G., "Oxidative stress signalling in Alzheimer's disease," Brain Research, 1000 (1-2). 32-39. 2004.

[7] Bustamante, J., Czerniczyniec, A., Cymeryng, C. and LoresArnaiz, S., "Age related changes from youth to adulthood in rat brain cortex: nitric oxide synthase and mitochondrial respiratory function," Neurochemical Research, 33 (7). 1216-1223. 2008.

[8] Atwood, C.S., Obrenovich, M.E., Liu, T., Chan, H., Perry, G., Smith, M.A. and Martins, R.N., "Amyloid- $\beta$ : a chameleon walking in two worlds: a review of the trophic and toxic properties of amyloid- $\beta, "$ Brain Research Reviews, 43 (1). 1-16. 2003.

[9] Lee, H.-g., Perry, G., Moreira, P.I., Garrett, M.R., Liu, Q., Zhu, X., Takeda, A., Nunomura, A. and Smith, M.A., "Tau phosphorylation in Alzheimer's disease: pathogen or protector," Trends in Molecular Medicine, 11 (4). 164-169. 2005.

[10] Mattson, M.P., "Roles of the lipid peroxidation product 4hydroxynonenal in obesity, the metabolic syndrome, and associated vascular and neurodegenerative disorders," Experimental Gerontology, 44 (10). 625-633. 2009.

[11] Lovell, M.A. and Markesbery, W.R., "Oxidative DNA damage in mild cognitive impairment and late-stage Alzheimer's disease," Nucleic Acids Research, 35 (22). 7497-7504. 2007.

[12] Lee, H.P., Zhu, X., Casadesus, G., Castellani, R.J., Nunomura, A., Smith, M.A., Lee, H-g. and Perry, G., "Antioxidant approaches for the treatment of Alzheimer's disease," Expert Review of Neurotherapeutics, 10 (7). 1201-1208. 2010.

[13] Lahiri, D.K., Farlow, M.R., Greig, N.H. and Sambamurti, K., "Current drug targets for Alzheimer's disease treatment," Drug Development Research, 56 (3). 267-281. 2002.

[14] Romano, C.S., Abadi, K., Repetto, V., Vojnov, A.A. and Moreno, S., "Synergistic antioxidant and antibacterial activity of rosemary plus butylated derivatives," Food Chemistry, 115 (2). 456-461. 2009.

[15] Lou, Z., Wang, H., Li, J., Chen, S., Zhu, S., Ma, C. and Wang, Z., "Antioxidant activity and chemical composition of the fractions 
from burdock leaves," Journal of Food Science, 75 (5). C413C419. 2010.

[16] Heo, H-J. and Lee, C-Y., "Epicatechin and catechin in cocoa inhibit amyloid- $\beta$ protein," Journal of Agricultural and Food Chemistry, 53 (5). 1445-1448. 2005.

[17] Chen, C-M., Lin, J-K., Liu, S-H. and Lin-Shiau, S-Y., "Novel regimen through combination of memantine and tea polyphenol for neuroprotection against brain excitotoxicity," Journal of Neuroscience Research, 86 (12). 2696-2704. 2008.

[18] Kwon, K.J., Kim, J.N., Kim, M.K., Lee, J., Ignarro, L.J., Kim, HJ., Shin, C.Y. and Han, S-H., "Melatonin synergistically increases resveratrol-induced heme oxygenase-1 expression through the inhibition of ubiquitindependent proteasome pathway: a possible role in neuroprotection," Journal of Pineal Research, 50 (2). 110123. 2011.

[19] Prior, R.L. and Gu, L., "Occurrence and biological significance of proanthocyanidins in the American diet," Phytochemistry, 66 (18). 2264-2280. 2005.

[20] Tarascou, I., Mazauric, J.-P., Meudec, E., Souquet, J.-M., Cunningham, D., Nojeim, S., Cheynier, V. and Fulcrand, H., "Characterisation of genuine and derived cranberry proanthocyanidins by LC-ESI-MS," Food Chemistry, 128 (3). 802-810. 2011.

[21] Ling, Z., Xie, B. and Yang, E., "Isolation, characterization, and determination of antioxidative activity of oligomeric procyanidins from the seedpod of Nelumbo nucifera Gaertn," Journal of Agricultural and Food Chemistry, 53 (7). 2441-2445. 2005.

[22] Gong, Y., Liu, L., Xie, B., Liao, Y., Yang, E. and Sun, Z., "Ameliorative effects of lotus seedpod proanthocyanidins on cognitive deficits and oxidative damage in senescence-accelerated mice," Behavioural Brain Research, 194 (1). 100-107. 2008.

[23] Xu, J., Rong, S., Xie, B., Sun, Z., Zhang, L., Wu, H., Yao, P., Hao, L. and Liu, L., "Procyanidins extracted from the lotus seedpod ameliorate age-related antioxidant deficit in aged rats," The Journals of Gerontology Series A-Biological Sciences and Medical Sciences, 65 (3). 236-241. 2010.

[24] Prakash, A. and Kumar, A., "Effect of $N$-acetyl cysteine against aluminium-induced cognitive dysfunction and oxidative damage in rats," Basic \& Clinical Pharmacology \& Toxicology, 105 (2). 98-104. 2009.

[25] Choy, K.H.C., Dean, O., Berk, M., Bush, A.I. and Buuse, M., "Effects of $\mathrm{N}$-acetyl cysteine treatment on glutathione depletion and a short-term spatial memory deficit in 2-cyclohexene-1-onetreated rats," European Journal of Pharmacology, 649 (1-3). 224228. 2010.

[26] Fu, A., Dong, Z. and Sun, M., "Protective effect of $N$-acetyl-Lcysteine on amyloid $\beta$-peptide-induced learning and memory deficits in mice," Brain Research, 1109. 201-206. 2006.

[27] Xu, J., Rong, S., Xie, B., Sun, Z., Zhang, L., Wu, H., Yao, P., Zhang, X., Zhang, Y. and Liu, L., "Rejuvenation of antioxidant and cholinergic systems contributes to the effect of procyanidins extracted from the lotus seedpod ameliorating memory impairment in cognitively impaired aged rats," European Neuropsychopharmacology, 19 (12). 851-860. 2009.

[28] Porter, L.J., Hrstich, L.N. and Chan, B.G., "The conversion of proanthocyanidins and prodelphinidins to cyaniding and delphinidin," Phytochemistry, 25 (1). 223-230. 1986.

[29] Xiao, J., Xie, B., Cao, Y., Wu, H., Sun, Z. and Xiao, D. "Characterization of oligomeric procyanidins and identification of quercetin glucuronide from lotus (Nelumbo nucifera Gaertn.) seedpod," Journal of Agricultural and Food Chemistry, 60 (11). 2825-2829. 2012.

[30] Xiao, J., Sui, Y., Li, S., Wu, Q., Yang, T., Xie, B. and Sun, Z., "Combination of proanthocyanidins extracted from lotus seedpod and L-cysteine ameliorates memory impairment induced by alcohol and scopolamine in mice," European Food Research and Technology, 236 (4). 671-679. 2013.

[31] Xu, J., Rong, S., Xie, B., Sun, Z., Deng, Q., Bao, W., Wang, D., Yao, P., Huang, F. and Liu, L., "Changes in the nitric oxide system contribute to effect of procyanidins extracted from the lotus seedpod ameliorating memory impairment in cognitively impaired aged rats," Rejuvenation Research, 14 (1). 33-43. 2011.

[32] Flood, J.F. and Cherkin, A., "Scopolamine effects on memory retention in mice: a model of dementia," Behavioral and Neural Biology, 45 (2). 169-184. 1986.

[33] Buccafusco, J.J., Terry, A.V., Webster, S.J., Martin, D., Hohnadel, E.J., Bouchard, K.A. and Warner, S.E., "The scopolamine-reversal paradigm in rats and monkeys: the importance of computerassisted operant-conditioning memory tasks for screening drug candidates," Psychopharmacology, 199 (3). 481-494. 2007.

[34] Egashira, N., Kurauchi, K., Iwasaki, K., Mishima, K., Orito, K., Oishi, R. and Fujiwara, M., "Schizandrin reverses memory impairment in rats," Phytotherapy Research, 22 (1). 49-52. 2008.

[35] Von Linstow Roloff, E., Harbaran, D., Micheau, J., Platt, B. and Riedel, G., "Dissociation of cholinergic function in spatial and procedural learning in rats," Neuroscience, 146 (3). 875-889. 2007.

[36] Gold, P.E., "Acetylcholine modulation of neural systems involved in learning and memory," Neurobiology of Learning and Memory, 80 (3). 194-210. 2003.

[37] Bartus, R.T., "On neurodegenerative diseases, models, and treatment strategies: lessons learned and lessons forgotten a generation following the cholinergic hypothesis," Experimental Neurology, 163 (2). 495-529. 2000.

[38] Dringen, R., "Metabolism and functions of glutathione in brain," Progress in Neurobiology, 62 (6), 649-671, 2000.

[39] Jovanovic, Z., "Mechanisms of neurodegeneration in Alzheimer's disease," Medicinski Pregled, 65 (7-8). 301-307. 2012.

[40] Sultana, R. and Butterfield, D.A., "Oxidative modification of brain proteins in Alzheimer's disease: perspective on future studies based on results of redox proteomics studies," Journal of Alzheimer's Disease, 33. S243-S251. 2013.

[41] Lee, M.R., Yun, B.S., Zhang, D.L., Liu, L., Wang, Z., Wang, C.L., Gu, L.J., Wang, C.Y., Mo, E.K., Ly, S.Y. and Sung, C., "Effect of aqueous antler extract on scopolamine-induced memory impairment in mice and antioxidant activities, "Food Science and Biotechnology, 19 (3). 655-661. 2010.

[42] Jovanovic, Z., "Antioxidative defense mechanisms in the aging brain "Archives of Biological Sciences, 66 (1). 245-252. 2014.

[43] Formigari, A., Santon, A. and Irato, P., "Efficacy of zinc treatment against iron-induced toxicity in rat hepatoma cell line H4-II-EC3," Liver International, 27 (1). 120-127. 2007.

[44] Shi, J., Liu, Q., Wang, Y. and Luo, G., "Coadministration of huperzine A and ligustrazine phosphate effectively reverses scopolamine-induced amnesia in rats," Pharmacology Biochemistry and Behavior, 96 (4). 449-453. 2010.

[45] Edwards, T.M. and Rickard, N.S., "New perspectives on the mechanisms through which nitric oxide may affect learning and memory processes," Neuroscience \& Biobehavioral Reviews, 31 (3). 413-425. 2007.

[46] Giove, T.J., Deshpande, M.M. and Eldred, W.D., "Identification of alternate transcripts of neuronal nitric oxide synthase in the mouse retina," Journal of Neuroscience Research, 87 (14). 3134-3142. 2009.

[47] Capettini, L.S.A., Cortes, S.F., Gomes, M.A., Silva, G.A.B., Pesquero, J.L., Lopes, M.J., Teixeira, M.M. and Lemos, V.S., "Neuronal nitric oxide synthase-derived hydrogen peroxide is a major endothelium-dependent relaxing factor," American Joural of Physiology-Heart and Circulatory Physiology, 295 (6). 25032511. 2008. 